\title{
Gastrin-releasing peptide promotes the growth of HepG2 cells via EGFR-independent ERK1/2 activation
}

\author{
XINQIU LI ${ }^{1}$, YUNFU LV ${ }^{1,3}$, AIHUA YUAN ${ }^{4}$, SHANYONG YI ${ }^{2}$, YONG MA $^{4}$ and ZONGFANG LI ${ }^{5}$ \\ ${ }^{1}$ Department of Hepatobiliary Surgery and ${ }^{2}$ Cancer Center, First Affiliated Hospital of Xi'an Jiaotong University, Xi'an; \\ ${ }^{3}$ Department of General Surgery, the People's Hospital of Hainan Province, Haikou; ${ }^{4}$ Department of General Surgery, \\ Nanjing First Hospital Affiliated to Nanjing Medical University, Nanjing; ${ }^{5}$ Department of General Surgery, \\ Second Affiliated Hospital of Xi'an Jiaotong University, Xi'an, P.R. China
}

Received January 22, 2010; Accepted April 8, 2010

DOI: $10.3892 /$ or_00000877

\begin{abstract}
Gastrin-releasing peptide (GRP) plays an important role in regulating tumor growth and migration. However, little is known about its role in human hepatocellular carcinoma (HCC) cells. This study explored the effect of GRP on the growth of HCC HepG2 cells and the underlying mechanisms. Expression of GRP and its cognate receptor (GRPR) were detected by immunocytochemisty, reverse transcriptionPCR and Western blotting and compared between two human HCC cell lines (HepG2 and MHCC97H) and a normal hepatic cell line (HL-7702). The effects of GRP on cell proliferation and signaling pathways were examined by Western blotting, MTT assay and flow cytometry. Both GRP and GRPR were overexpressed in HepG2 and MHCC97H cells. GRP activated MAPK/ERK1/2 in HepG2 cells, leading to enhanced proliferation, reduced apoptosis and accelerated cell cycle progression. The effect of GRP on ERK1/2 was effectively attenuated by the GRPR antagonist PD176252 or MEK inhibitor U0126, but not by the TNF- $\alpha$ protease inhibitor TAPI-1 or the EGFR tyrosine kinase inhibitor PD153035. The effect of GRP on the growth of HepG2 cells was significantly attenuated by PD176252 or U0126. GRP serves as a mitogen for HepG2 and MHCC97H cells. GRP promotes the growth of HepG2 cells through interaction with GRPR co-expressed in tumor cells, and subsequently activates MAPK/ERK1/2 via EGFRindependent mechanisms.
\end{abstract}

\section{Introduction}

Accumulating evidence has indicated that neuropeptides play important roles in regulating tumor growth and migration. For example, extensive studies have shown that mammalian

Correspondence to: Dr Yunfu Lv, Department of Hepatobiliary Surgery, First Affiliated Hospital of Xi'an Jiaotong University, 277 West Yanta Road, 710061 Xi'an, P.R. China

E-mail: tougaolxq@yahoo.cn

Key words: gastrin-releasing peptide, gastrin-releasing peptide receptor, mitogen-activated protein kinase, extracellular signalregulated kinase $1 / 2$, hepatocellular carcinoma bombesin-like gastrin-releasing peptide (GRP) binds to its cognate receptor (GRPR) and exerts various biological effects in tumors $(1,2)$. In particular, GRP serves as a potent mitogen for various types of tumor including small cell lung, pancreatic, prostate, renal, breast and colon cancers (1-7). Furthermore, treatment with GRP antibody was found to lead to significant anti-proliferative effects, indicating that GRP is an autocrine growth factor and GRPR may be a drug target for tumor imaging and anti-tumor therapy (8-14).

Several studies have demonstrated a stimulatory role of GRPR for the mitogen-activated protein kinase/extracellular signal-regulated kinase 1/2 (MAPK/ERK1/2) signaling pathway in a number of cancer cells (15-18). The mechanisms through which GRPR activated MAPK/ERK1/2 in tumor cells were not fully clarified, but appeared to vary in different types of tumor cells. For example, GRP was found to interact with GRPR in tumor cells to activate the TNF- $\alpha$ convertase (TACE), which in turn switched on the MAPK/ERK1/2 signaling pathway by shedding ligands for the epidermal growth factor receptors (EGFR) $(17,18)$.

Experimental animal studies demonstrated that rat hepatocellular tumor cells also produced functional GRP and GRPR molecules that appeared to promote hepatocarcinogenesis (19). However, little is known about the role of GRP and GRPR in human hepatocellular carcinoma (HCC). HCC is a highly aggressive and the most common malignancy in liver with poor prognosis. Previously, it has been suggested that the poor prognosis of HCC may be associated with elevated MAPK/ERK1/2 activities in tumor cells (20-22). In the present study, we compared the expressions of GRP and GRPR in human hepatoma HepG2 and MHCC97H with those in normal hepatic HL-7702 cells. Furthermore, we investigated the influence of GRP on the growth of HepG2 cells in correlation with GRPR and the intracellular activity of MAPK/ ERK1/2. Our observations provide important insights into the function and mechanism of GRP in the regulation of tumor cell growth.

\section{Materials and methods}

Chemicals and antibodies. Human GRP (sequence: VPLPAGGGTVLTKMYPR GNHWAVGHLM-NH2) was obtained from Sigma-Aldrich (St. Louis, USA). Antibodies 
against GRP, GRPR, TACE and phospho-TACE (T735) were obtained from Abcam (Cambridge, UK). Antibodies against EGFR and phospho-EGFR (Tyr1086) were obtained from Upstate Biotech (NY, USA). Antibody against p44/42MAPK (ERK1/2) was obtained from Santa Cruz Biotechnology (Santa Cruz, CA, USA) and antibody against phosphoERK1/2 (T202/Y204) was obtained from R\&D Systems (USA). Specific secondary antibodies, EGFR tyrosine kinase inhibitor PD153035, and MEK inhibitor U0126 were obtained from Calbiochem-Novabiochem (San Diego, CA, USA). The non-peptide gastrin releasing peptide receptor antagonist (S)-N-[[1-(5-Methoxy-2-pyridinyl)cyclohexyl]methyl]-amethyl-a-[[[-(4-nitrophenyl)amino]carbonyl] amino-1Hindole-3-propanamide (PD 176252) was obtained from Tocris Bioscience (Bristol, UK). The TNF- $\alpha$ protease inhibitor-1 (TAPI-1, N-(R)-[2-(Hydroxyaminocarbonyl) methyl]-4methylpentanoyl-L-naphthylalanyl-Lalanine, 2-aminoethyl Amide) was obtained from Merck Biosciences (UK).

Cell culture and treatment. Human normal liver cell line HL-7702 and human hepatoma cell line HepG2 were obtained from ATCC (Rockville, MD, USA), and the highly invasive human hepatocellular carcinoma cell line MHCC97H was obtained from Liver Cancer Institute of Zhongshan Hospital, Fudan University (Shanghai, China). HL-7702 and MHCC97H cells were maintained and propagated in Dulbecco's modified Eagle's medium (DMEM) with high glucose supplemented with $15 \%$ fetal bovine serum (HyClone, USA). HepG2 cells were maintained and propagated in RPMI-1640 supplemented with $10 \%$ fetal bovine serum. All cultured cells were maintained at $37^{\circ} \mathrm{C}$ in a humidified atmosphere containing $5 \%$ $\mathrm{CO}_{2}$ and experiments were done using 70-80\% confluent cultures.

Immunocytochemistry. The expression of GRP and GRPR in the afore-mentioned three cell lines was assessed by immunocytochemisty. Cells plated on glass cover slips were fixed by $4 \%$ paraformaldehyde, and permeabilized with $0.5 \%(\mathrm{v} / \mathrm{v})$ Triton. Cells were washed with PBS, treated with ice-cold methanol for $20 \mathrm{~min}$, and pre-blocked for $30 \mathrm{~min}$ with sheep serum at $37^{\circ} \mathrm{C}$. Cells were subsequently washed twice with PBS, and incubated with anti-GRP and anti-GRPR (1:100) overnight at $4^{\circ} \mathrm{C}$. After PBS wash, cells were incubated with secondary goat anti-rabbit immunoglobulin conjugated with biotin for $60 \mathrm{~min}$ at room temperature, followed by incubation with streptavidin-peroxidase complex for $30 \mathrm{~min}$. The peroxidase reaction products were visualized by incubation with $0.05 \mathrm{M}$ Tris- $\mathrm{HCl}$ buffer ( $\mathrm{pH} 7.6)$ containing $20 \mathrm{mg}$ 3,3-diaminobendizine (DAB). The slides were counterstained for nuclei with hematoxylin and mounted with gum.

Reverse transcription-PCR. Total cellular RNA of HL-7702, HepG2 and MHCC97H cells was isolated using TRIzol reagent according to the manufacturer's protocol (Invitrogen, USA). RNA concentration was measured by GeneQuant II (Pharmacia, Uppsala, Sweden) at $260 \mathrm{~nm}$. Equal amount of total RNAs were used for reverse transcription reaction and cDNA synthesis in accordance with the manufacturer's instructions (Invitrogen). The PCR products were electrophoresed on a $2 \%$ agarose gel and visualized by ethidium bromide staining. The molecular weight was derived based on a molecular weight marker and B-actin was used as the loading control. Results were quantitated using densitometry and Image J software (version 1.34s; by Wayne Rasband, NIH, USA). The following primers were used to determine the mRNA expression for GRP, GRPR and $\beta$-actin. GRP forward, 5'-GGACCGTGCTGACCAAGATG-3'; reverse, 5'-AAGTTGCTGCTATCCTCTGAATCC-3'; GRPR forward, 5'-GCCTGTTCGCCTTCTGCTG-3'; reverse, 5'-TCCTTCC AGTGCTGTGAGACC-3'; ß-actin forward, 5'-GTCCTCTC CCAAGTCCACAC-3'; reverse, 5'-GGGAGACCAAAAG CCTTCAT-3'. The PCR conditions were: $94^{\circ} \mathrm{C}$ for $2 \mathrm{~min}$ followed by 35 cycles $\left(94^{\circ} \mathrm{C}\right.$ for $30 \mathrm{sec}, 58^{\circ} \mathrm{C}$ for $30 \mathrm{sec}$ and $72^{\circ} \mathrm{C}$ for $1 \mathrm{~min}$ ), and a final extension at $72^{\circ} \mathrm{C}$ for $10 \mathrm{~min}$.

Western blot analysis. Cells were washed twice in PBS, lysed in ice-cold radioimmune precipitation (RIPA) buffer, and then centrifuged for $10 \mathrm{~min}$ at $4^{\circ} \mathrm{C}$. Supernatants were collected and protein concentrations were determined and adjusted to $2 \mathrm{mg} / \mathrm{ml}$ using a Bio-Rad protein assay kit (Bio-Rad, USA). Cell lysates were mixed with $3 \mathrm{X}$ Laemmli buffer and heated for $5 \mathrm{~min}$ at $95^{\circ} \mathrm{C}$. Sample proteins were then resolved by SDS-PAGE (8 or $10 \%$ polyacrylamide gels), transferred to PVDF membrane (Immobilon ${ }^{\mathrm{TM}}$; Millipore) by electroblotting. The membrane was blotted with $10 \%$ non-fat milk, washed in TBS/Tween and incubated with primary rabbit polyclonal antibodies overnight at $4^{\circ} \mathrm{C}$. After washing with TBS/Tween, they were incubated with secondary antibody (horseradish peroxidase conjugated $\mathrm{IgG}$ ) for $60 \mathrm{~min}$ at room temperature. Membranes were washed again with TBS/Tween before detection using the ECL detection system (Amersham Pharmacia, USA). The dilutions for the primary antibodies were as follows: anti-GRP, 1:400; anti-GRPR, 1:400; antiTACE, 1:800; anti-phospho-TACE, 1:800; anti-EGFR, 1:800; anti-phospho-EGFR, 1:800; anti-ERK1/2, 1:800; antiphospho-ERK1/2, 1:800; anti-ß-actin, 1:800; Secondary antibody was used at a dilution of 1:10000. Results were quantitated using densitometry and Image $\mathbf{J}$ software (version 1.34s; by Wayne Rasband, NIH, Bethesda, MD, USA).

Cell proliferation assay. Proliferation of HL-7702, HepG2 and MHCC $97 \mathrm{H}$ cells was investigated through MTT [3-(4,5dimethylthiazol-2-yl)-2, 5-diphenyl-tetrazolium bromide; Sigma] assay. Briefly, $1 \times 10^{4}$ cells per well were plated into flat-bottomed 96-well plates (Costar, Corning, USA). After $24 \mathrm{~h}$, cells were serum-starved overnight. The next day, cells were stimulated by different concentrations of GRP in the absence or presence of inhibitors for different times. Control wells were treated with $0.1 \%$ dimethyl sulphoxide (DMSO) alone. Subsequently, $20 \mathrm{ml}$ MTT (5 g/l) was added to each well and incubated for an additional $4 \mathrm{~h}$. Plates were centrifuged for $5 \mathrm{~min}$ at $1000 \mathrm{rpm}$, and the medium was carefully discarded. The formed formazan crystals were dissolved in $100 \mu \mathrm{l}$ of DMSO, and absorbance (A) was read at $570 \mathrm{~nm}$ using a spectrophotometer. The percentage of viable cells was calculated as follows: (A of experimental group/A of control group) x $100 \%$.

Cell cycle and apoptosis analysis. HepG2 cells were serumstarved overnight. The synchronized cells were then treated 


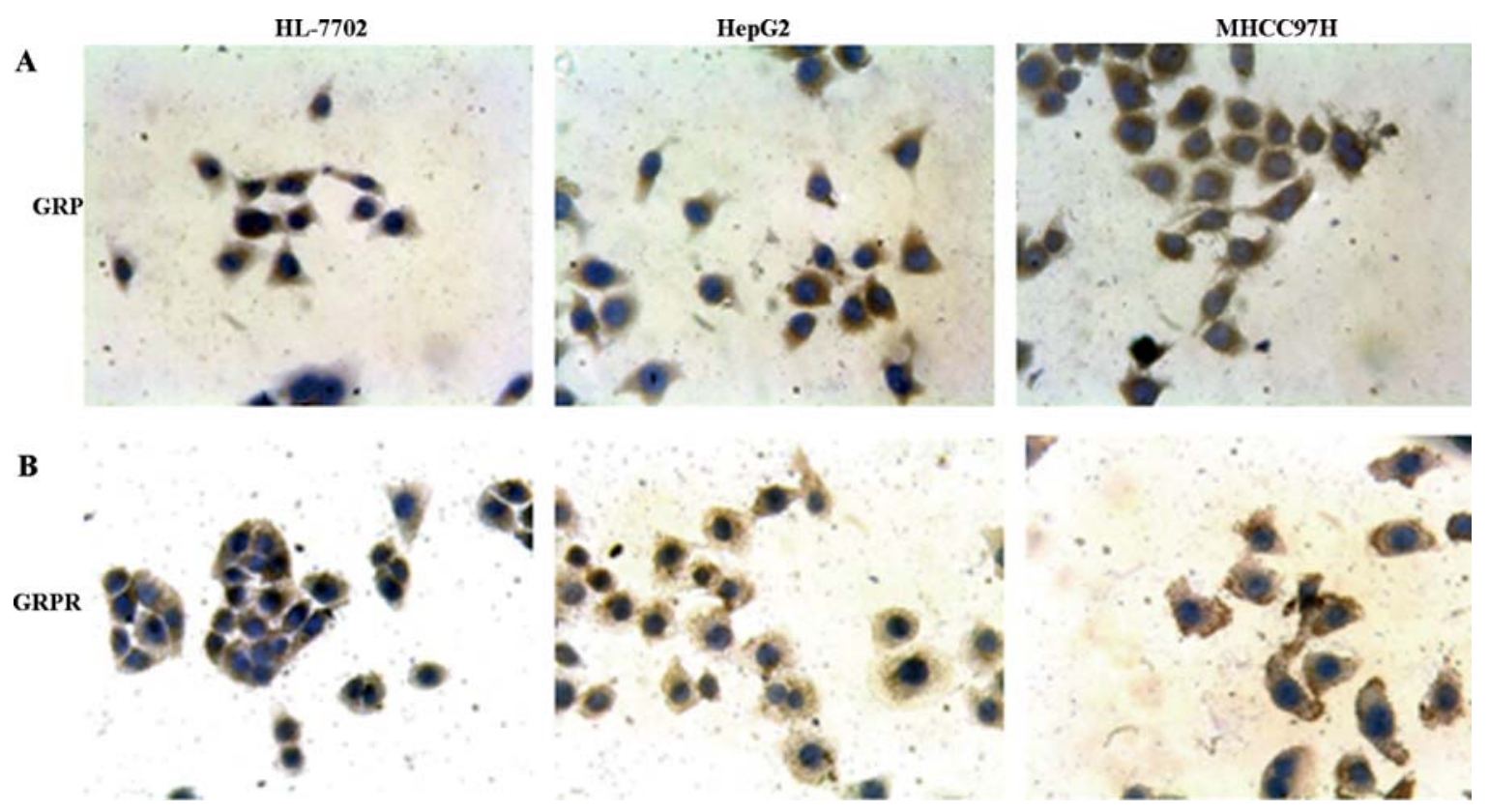

Figure 1. Immunochemistry analysis of the expression of gastrin-releasing peptide (GRP) and its cognate receptor (GRPR) in HL-7702, HepG2 and MHCC97H Cells. The expression of GRP and GRPR in HL-7702, HepG2 and MHCC97H cells was assessed by immunocytochemisty. Similar results were obtained in three independent experiments and representative results are shown. Both GRP and GRPR expressed at higher levels (luteotestaceously stained) in the cytoplasma and at the plasma membrane of HepG2 and MHCC97H cells than in HL-7702 cells.

for the indicated times, and harvested by trypsinization. PBS washed cells were fixed in ice-cold $80 \%$ ethanol, washed, and resuspended in $1 \mathrm{ml}$ PBS. Samples were treated with $10 \mu \mathrm{l}$ RNase A $(21 \mathrm{mg} / \mathrm{ml})$, and stained with $5 \mu 1$ propidium iodide (PI) at $1 \mathrm{mg} / \mathrm{ml}$ for $30 \mathrm{~min}$ at room temperature in the dark. The stained cells were analyzed by flow cytometry (BD LSRII System, BD Biosciences, USA). To evaluate the cell cycle distribution of apoptotic cells, treated cells were washed in PBS, and resuspended in $100 \mathrm{ml}$ binding buffer containing FITC-conjugated annexin V (Becton-Dickinson, USA) and PI to discriminate apoptotic cells from live cells and necrotic cells. Annexin V(+)/PI(-) and Annexin V(+)/PI(+) represented cells in early apoptosis and late apoptosis/necrosis, respectively.

Statistical analysis. Results were expressed as the mean \pm SEM of three separate experiments and analyzed by the computer program SPSS 13.0 for Windows (SPSS Inc., USA), using one-way analysis of variance (ANOVA) followed by the Tukey's t-test. P-values $<0.05$ were considered statistically significant.

\section{Results}

Overexpression of GRP and GRPR in HepG2 and MHCC97H cells. We first examined GRP and GRPR expression in both tumor and normal cell lines. As shown in Fig. 1, immunocytochemisty analysis revealed readily detectable GRP and GRPR expression in the three cell lines HL-7702, HepG2 and MHCC97H. Importantly, elevated expression was observed in the two tumor cell lines HepG2 and MHCC97H compared to the human normal liver cell line HL-7702 (Fig. 1). Likewise, RT-PCR analysis demonstrated that both GRP mRNA and
GRPR mRNA levels were elevated in the two tumor cell lines compared to that in HL-7702. Specifically, GRP mRNA showed 3.21-fold higher levels in HepG2 and 3.70-fold in MHCC97H versus HL-7702, and GRPR mRNA was 3.52fold higher in HepG2 and 3.61-fold in MHCC97H than that of HL-02 (Fig. 2A). Overexpression of GRP and GRPR in tumor cells was further confirmed at the protein level by Western blotting. Levels of GRP peptides and GRPR proteins were enhanced in HepG2 (1.71- and 2.10-fold, respectively) and in MHCC97H (1.82- and 2.92-fold, respectively) versus HL-7702 (Fig. 2B).

Stimulatory effects of GRP on the proliferation of HepG2 and MHCC $97 H$ cells. Next we investigated the physiological significance of the elevated expression of GRP in tumor cells. MTT assays were carried out to investigate the effects of GRP on cell proliferation. The proliferation of HL-7702 cells was not significantly influenced; however, GRP appeared to markedly enhance the proliferation of HepG2 and MHCC97H cells in a dose-dependent manner (Fig. 3A). Specifically, stimulatory effects on proliferation of HepG2 and $\mathrm{MHCC} 97 \mathrm{H}$ cells was first observed with $0.1 \mathrm{nM}$ of GRP $(115 \pm 6 \%$ and $117 \pm 3 \%$ of control, respectively; $\mathrm{P}<0.05)$ and peaked at $100 \mathrm{nM}$ of GRP with the maximum increase of proliferation ( $167 \pm 8 \%$ and $173 \pm 4 \%$ of control, respectively; $\mathrm{P}<0.01$ ). No significant influence of GRP was detected at concentrations lower than $0.1 \mathrm{nM}$, and no greater effect of GRP on the proliferation of HepG2 and MHCC97H cells was observed at concentrations higher than $100 \mathrm{nM}$. To further investigate the time-dependence of GRP effects, we incubated cells with $100 \mathrm{nM}$ of GRP for $0,12,24,48$ and $96 \mathrm{~h}$. Whereas GRP exerted virtually no effect on the proliferation of HL-7702 cells, time-dependent stimulatory effects on HepG2 and 


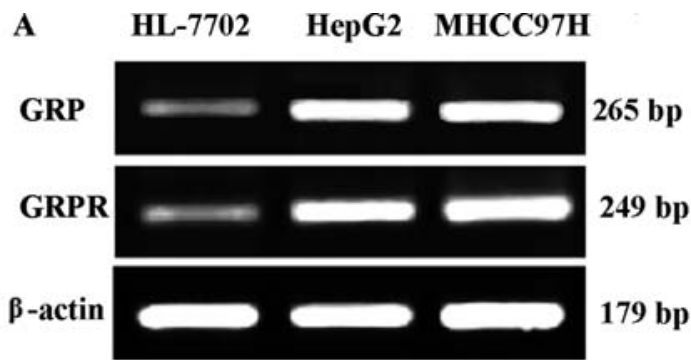

口HL-7702 $\square$ HepG2 目MHCC97H
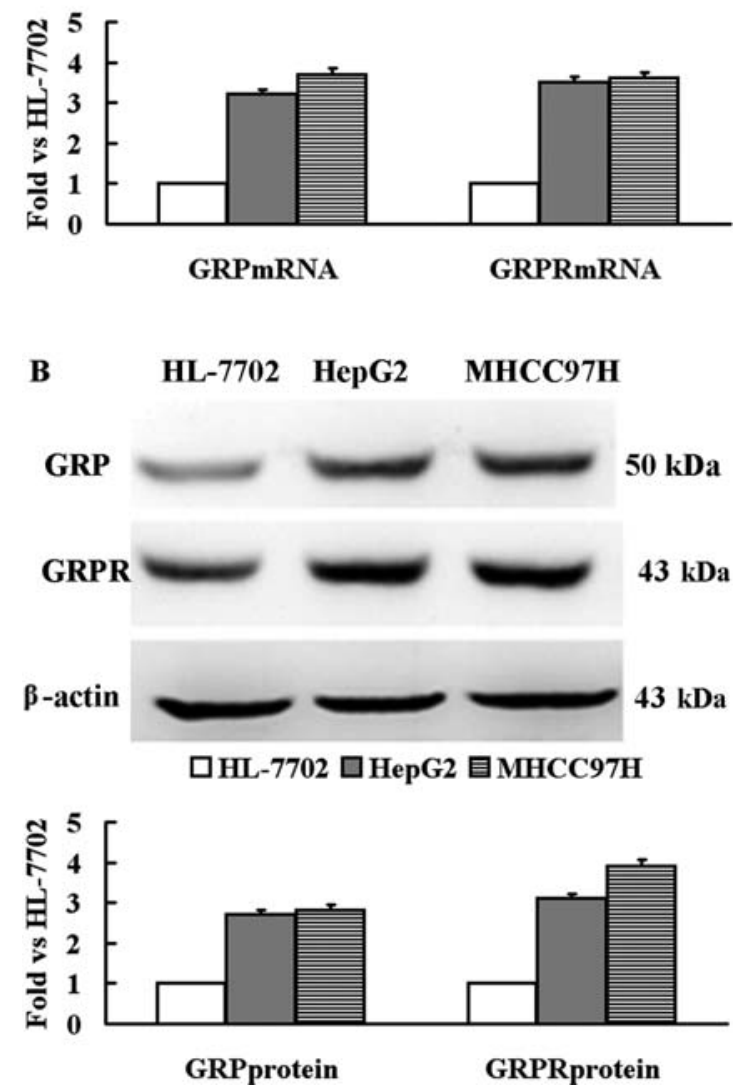

Figure 2. Reverse transcription-PCR and Western blotting analysis of the expression of GRP and GRPR in HL-7702, HepG2 and MHCC97H cells. Results for three independent experiments are shown. The normalized data against the control (HL-7702) are presented as mean \pm SEM. (A) GRP and GRPR mRNA expression detected by RT-PCR. (B) GRP and GRPR protein expression detected by Western blotting.

MHCC97H cell proliferation by GRP were observed (Fig. 3B). From $12 \mathrm{~h}(126 \pm 8 \%$ and $132 \pm 6 \%$ of control, respectively; $\mathrm{P}<0.01)$ to $48 \mathrm{~h}(163 \pm 3 \%$ and $172 \pm 2 \%$ of control, respectively; $\mathrm{P}<0.01)$, increasing stimulatory effects of GRP on HepG2 and MHCC97H cells were observed, and longer incubation time did not lead to greater stimulatory effect.

GRP activated MAPK/ERK1/2 in HepG2 cells. Previous evidence indicated that human HCC exhibited high levels of MAPK/ERK1/2 and enhanced activity of MAPK/ERK1/2 in tumor cells predicted poor prognosis $(21,22)$. To explore a possible correlation of GRP with the activity of MAPK/ ERK1/2 in HepG2 cells, we examined the stimulatory effects of GRP on ERK1/2. GRP (100 nM) was added to HL-7702 and HepG2 cells and the levels of phosphorylated-ERK1/2 (pERK1/2) were determined by Western blotting. The addition
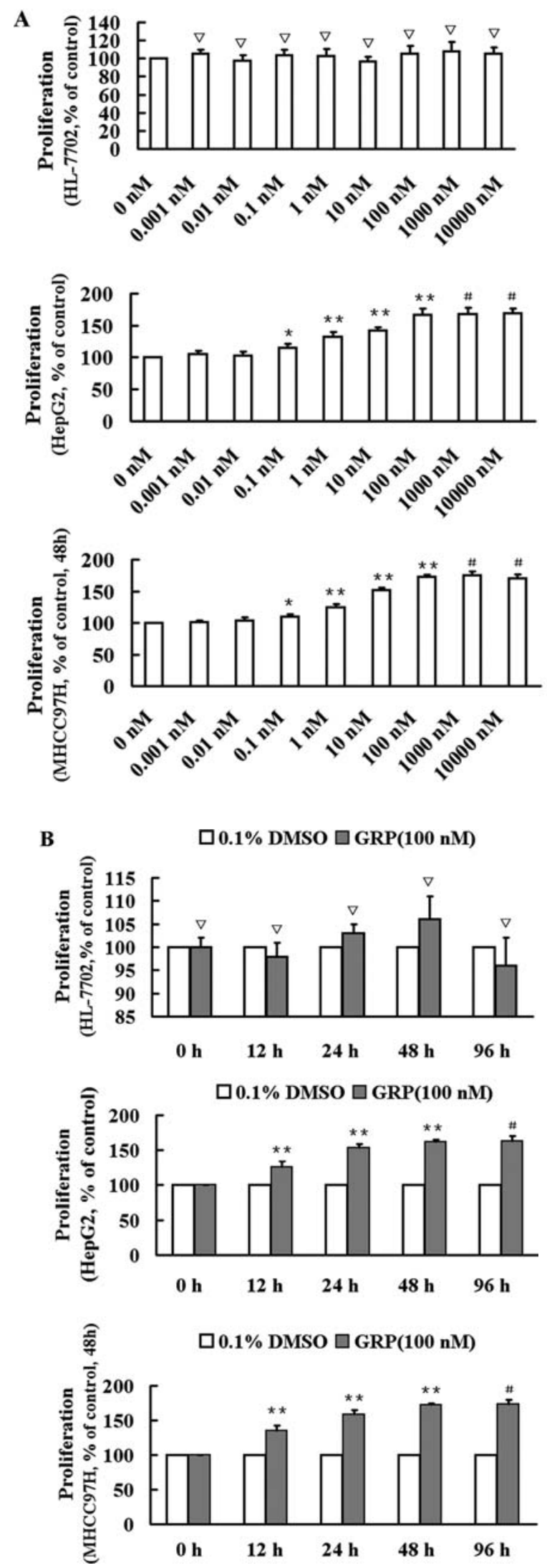

Figure 3. Proliferation-promoting effects of GRP on HepG2 and MHCC97H cells. HL-7702, HepG2 and MHCC97H cells were serum-starved overnight before treatment and the cell proliferation was measured via the MTT method. Results from three independent experiments are presented as mean percent of control \pm SEM. (A) Cells were stimulated by different concentrations of GRP for $48 \mathrm{~h} .{ }^{\nabla} \mathrm{P}>0.05$ vs. $0 \mathrm{nM} .{ }^{*} \mathrm{P}<0.05,{ }^{* *} \mathrm{P}<0.01$ vs. control $(0 \mathrm{nM})$. ${ }^{\#} \mathrm{P}>0.05$ vs. $100 \mathrm{nM}$. (B) Cells were stimulated by $100 \mathrm{nM}$ of GRP for $0,12,24,48$ and $96 \mathrm{~h} .{ }^{\nabla} \mathrm{P}>0.05,{ }^{*} \mathrm{P}<0.05,{ }^{* *} \mathrm{P}<0.01$ vs. control $(0.1 \%$ DMSO). ${ }^{*} \mathrm{P}>0.05$ vs. $48 \mathrm{~h}$. 
A
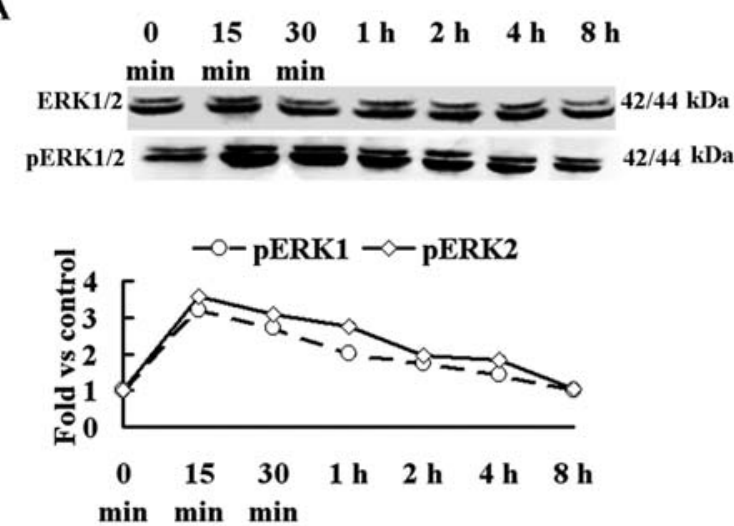
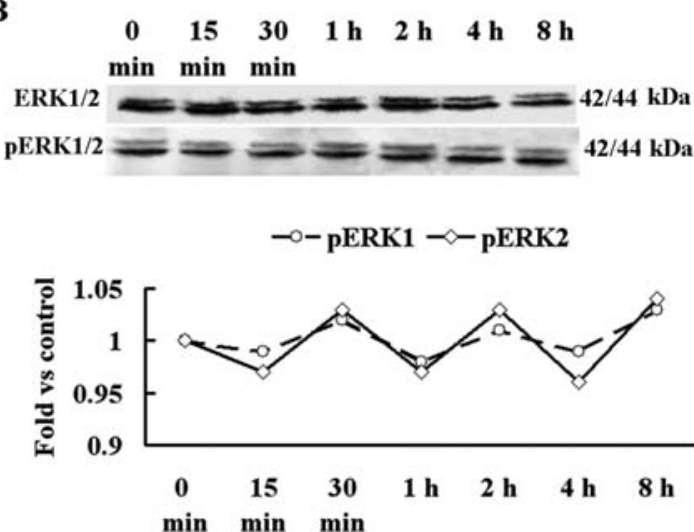

Figure 4. GRP transiently activated MAPK/ERK1/2 in HepG2 cells. HL-7702 and HepG2 cells were serum-starved overnight and then incubated with 100 nM of GRP for 15 and $30 \mathrm{~min}$, and 1, 2, 4 and $8 \mathrm{~h}$. The levels of ERK1/2 and phosphorylated-ERK1/2 were determined by Western blotting using specific antibody against ERK1/2 and phosphorylated-ERK1/2. Results from three independent experiments are shown. The results are presented as fold changes versus control (0 min). (A) The expression of ERK1/2 and phosphorylated-ERK1/2 in HepG2 cells. (B) The expression of ERK1/2 and phosphorylatedERK1/2 in HL-7702 cells. Similar results were obtained in three independent experiments.

of GRP to HepG2 cells resulted in an immediate activation of ERK1/2. As early as $15 \mathrm{~min}$ after the administration of GRP, pERK1/2 was increased by an average of 2.21- and 2.56-fold. In contrast, HL-7702 cells, which weakly express pERK1/2, were not affected by GRP. However, the activation of ERK1/2 in HepG2 cells was transient, and gradually declined to baseline level within $8 \mathrm{~h}$ (Fig. 4).

MAPK/ERK1/2 activation by GRP in HepG2 required GRPR, but not EGFR. To confirm the stimulatory effect of GRP on ERK1/2 was mediated by GRPR, cells were treated with the non-peptide gastrin-releasing peptide receptor antagonist PD176252 or the MEK inhibitor U0126 before GRP treatment. To rule out the possibility that GRPR activation may lead to TACE-dependent transactivation of EGFR which may in turn activate ERK1/2 $(17,18)$, the TACE inhibitor TNF- $\alpha$ protease inhibitor-1 (TAPI-1) and the inhibitor of EGFR tyrosine kinase PD153035 were also applied before GRP administration $(17,21,22)$. HepG2 cells were first stimulated with either $0.1 \%$ DMSO (control), or $100 \mathrm{nM}$ of GRP with or without $1 \mu \mathrm{M}$ of TAPI-1. The levels of pTACE, pEGFR and pERK1/2 were determined by Western blotting. As shown in Fig. 5A, the levels of pTACE, pEGFR, pERK1 and pERK2 were all increased by $100 \mathrm{nM}$ of GRP (1.98-, 1.55-, 3.21- and 3.56-fold over control, respectively). The presence of TAPI-1 attenuated the effect of GRP on pTACE and pEGFR, but not on pERK1/2 (Fig. 5A). We next stimulated HepG2 cells with GRP (100 nM) with or without PD176252 (5 $\mu \mathrm{M})$, PD153035 (25 $\mu \mathrm{M})$ or U0126 $(10 \mu \mathrm{M})$. GRP-induced phosphorylation of ERK1/2 was attenuated by PD176252 and U0126, but not by PD153035 (Fig. 5B).

The growth-promoting effect of GRP was mainly mediated by GRPR-induced activation of MAPK/ERK1/2. To confirm the ERK1/2-dependent effect of GRP on HepG2 cells, we incubated HepG2 cells with $100 \mathrm{nM}$ of GRP in the presence or absence of the GRPR antagonist PD176252 $(5 \mu \mathrm{M})$ or the MEK inhibitor U0126 $(10 \mu \mathrm{M})$. Cell proliferation was evaluated by MTT assay, and the apoptotic cells and cell cycle progression were analyzed by flow cytometry. GRP-
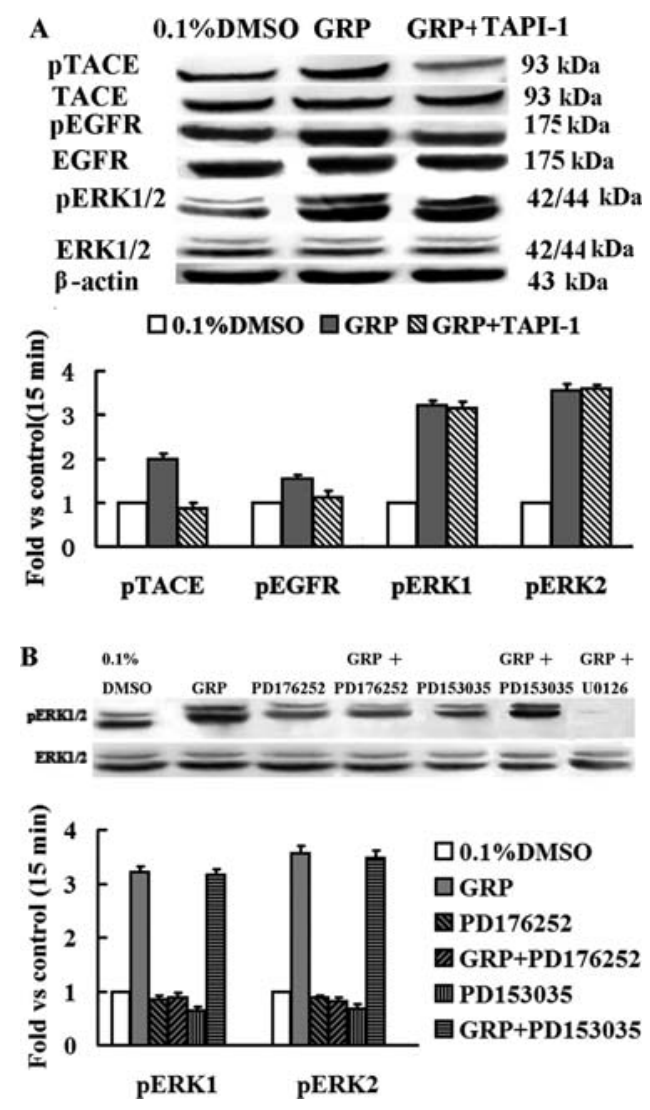

Figure 5. MAPK/ERK1/2 activation by GRP required GRPR, but not EGFR. HepG2 cells were serum-starved overnight and then treated with GRP $(100 \mathrm{nM})$ in the presence or absence of different inhibitors. Results from three independent experiments are shown. (A) HepG2 cells were stimulated by $0.1 \%$ DMSO (control), GRP (100 nM), GRP (100 nM) + TAPI-1 $(1 \mu \mathrm{M})$. TAPI- 1 was added 30 min before GRP. At 15 min after GRP administration, the expressions of TACE, phosphorylated-TACE, EGFR, phosphorylatedEGFR, ERK1/2, phosphorylated-ERK1/2 were detected by Western blotting using specific antibodies. The results were presented as folds of control. (B) HepG2 cells were stimulated by $0.1 \%$ DMSO (control), GRP (100 nM), PD176252 $(5 \mu \mathrm{M}), \operatorname{GRP}(100 \mathrm{nM})+\operatorname{PD} 176252(5 \mu \mathrm{M}), \operatorname{PD} 153035(25 \mu \mathrm{M})$, $\operatorname{GRP}(100 \mathrm{nM})+\operatorname{PD} 153035(25 \mu \mathrm{M})$ and GRP $(100 \mathrm{nM})+\mathrm{U} 0126(10 \mu \mathrm{M})$, respectively. PD176252, PD153035 and U0126 were added $30 \mathrm{~min}$ before adding GRP. At 15 min after GRP stimulation, the expression of ERK1/2 and phosphorylated-ERK1/2 were detected by Western blotting using specific antibodies. The data are presented as folds of control. 


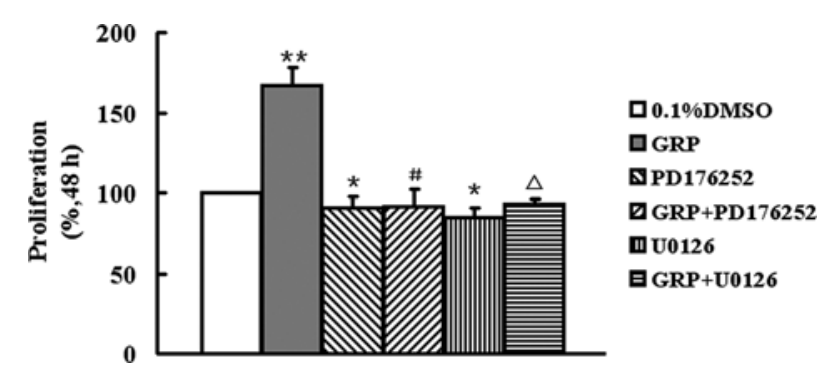

Figure 6. GRP promotes the growth of HepG2 cells by a MAPK/ERK1/2dependent mechanism. HepG2 cells were serum-starved overnight and then stimulated by $0.1 \%$ DMSO (control), GRP $(100 \mathrm{nM})$, PD176252 $(5 \mu \mathrm{M})$, GRP $(100 \mathrm{nM})+\mathrm{PD} 176252(5 \mu \mathrm{M}), \mathrm{U} 0126(10 \mu \mathrm{M})$ and GRP $(100 \mathrm{nM})+$ U0126 $(10 \mu \mathrm{M})$, respectively. PD176252 and U0126 were added $30 \mathrm{~min}$ before adding GRP. At $48 \mathrm{~h}$ after GRP administration, cell proliferation was evaluated using the MTT assay and the results are presented as percent of control. ${ }^{*} \mathrm{P}<0.05,{ }^{* *} \mathrm{P}<0.01$ vs. control ( $0.1 \%$ DMSO). ${ }^{*} \mathrm{P}>0.05$ vs. PD176252. $\triangle \mathrm{P}<0.05$ vs. U0126.
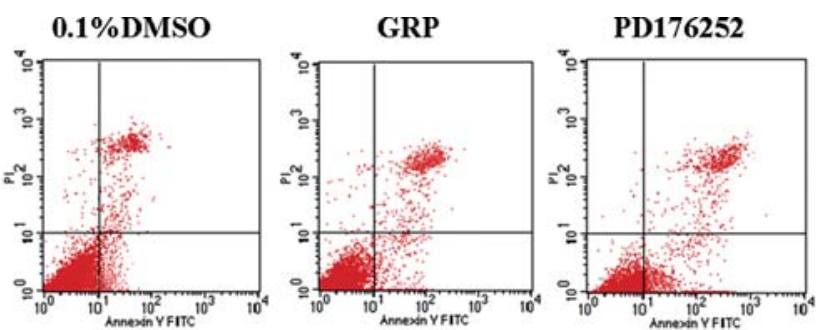

GRP+PD176252
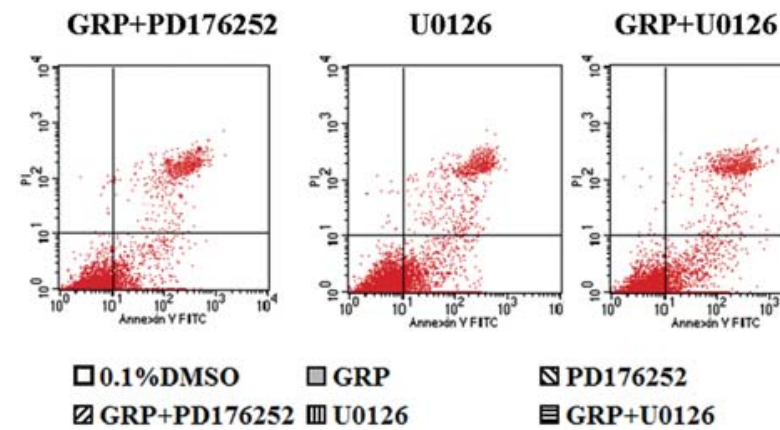

ब PD176252 ש GRP+PD176252 四 U0126

目 GRP+U0126

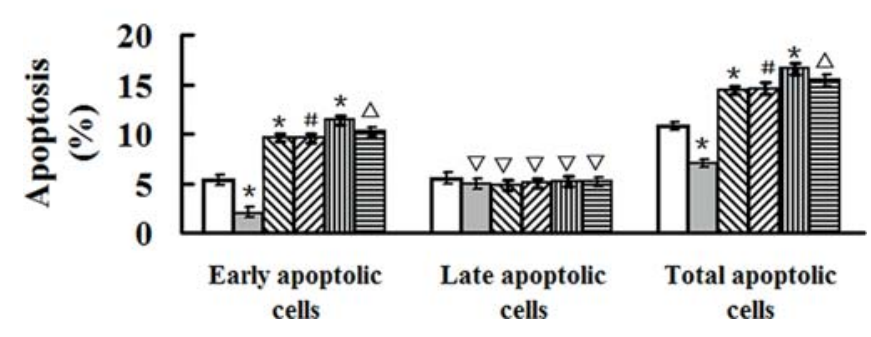

Figure 7. GRP inhibits the apoptosis of HepG2 cells. HepG2 cells were serum-starved overnight and then stimulated by $0.1 \%$ DMSO (control), GRP $(100 \mathrm{nM})$, PD176252 $(5 \mu \mathrm{M})$, GRP (100 nM) + PD176252 (5 $\mu \mathrm{M})$, U0126 $(10 \mu \mathrm{M})$ and GRP $(100 \mathrm{nM})+\mathrm{U} 0126(10 \mu \mathrm{M})$, respectively. PD176252 and U0126 were added $30 \mathrm{~min}$ before adding GRP. At $24 \mathrm{~h}$ after GRP treatment, apoptotic cells were evaluated by flow cytometry. Annexin V(+)/PI(-) and Annexin $\mathrm{V}(+) / \mathrm{PI}(+)$ represent the cells in early apoptosis and late apoptosis/ necrosis, respectively. The results are presented as percent of control. ${ }^{*} \mathrm{P}<0.01$ vs. $0.1 \%$ DMSO. ${ }^{\#} \mathrm{P}>0.05$ vs. PD176252. ${ }^{4} \mathrm{P}<0.05$ vs. U0126. ${ }^{\nabla} \mathrm{P}>0.05$ vs. $0.1 \%$ DMSO.

enhanced proliferation of HepG2 cells was abolished by PD176252 (GRP+PD176252 vs. PD176252: $92 \pm 10 \%$ vs. $91 \pm 7 \%$ of control; $P>0.05)$ and significantly compromised by $\mathrm{U} 0126$ (GRP+ U0126 vs. U0126, $85 \pm 6 \%$ vs. $93 \pm 3 \%$ of
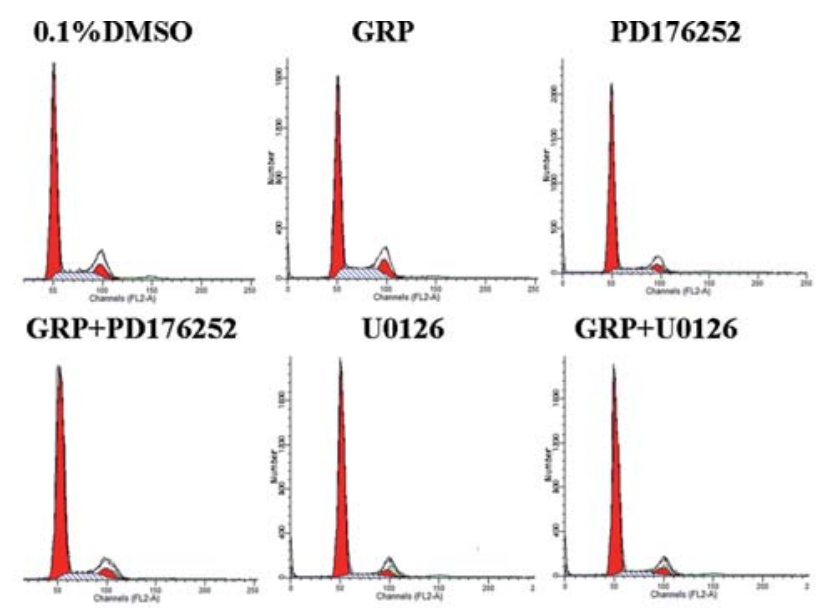

GRP+U0126

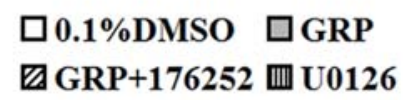

\$PD176252

目 GRP+U0126

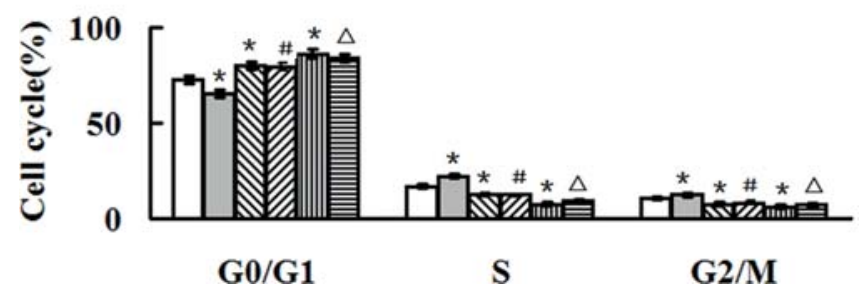

Figure 8. Stimulatory effects of GRP on cell cycle progression of HepG2 cells. HepG2 cells were serum-starved overnight and then stimulated by $0.1 \%$ DMSO (control), GRP (100 nM), PD176252 (5 $\mu \mathrm{M})$, GRP (100 nM) + PD176252 $(5 \mu \mathrm{M}), \mathrm{U} 0126(10 \mu \mathrm{M})$ and GRP $(100 \mathrm{nM})+\mathrm{U} 0126(10 \mu \mathrm{M})$, respectively. PD176252 and U0126 were added $30 \mathrm{~min}$ before adding GRP. At $48 \mathrm{~h}$ after GRP treatment, cells were stained with propidium iodide at $1 \mathrm{mg} / \mathrm{ml}$ for $30 \mathrm{~min}$. Cell cycle distributions were also evaluated by flow cytometry and the results are presented as percent of control. ${ }^{*} \mathrm{P}<0.05$ vs. control (0.1\% DMSO). ${ }^{*} \mathrm{P}>0.05$ vs. PD176252. $\triangle \mathrm{P}<0.05$ vs. U0126.

control; P<0.05) (Fig. 6). Furthermore, GRP significantly reduced the early and total apoptosis (AP) of HepG2 cells (GRP vs. $0.1 \%$ DMSO: early AP: $2.01 \pm 0.55 \%$ vs. $5.33 \pm 0.52 \%$; total AP: $6.98 \pm 0.48 \%$ vs. $10.42 \pm 0.39 \%$; $<<0.01$ ). The percentage of apoptotic cells was significantly increased in the samples stimulated by PD176252 or U0126 (early and total AP; $\mathrm{P}<0.01$ ); conversely, GRP-dependent apoptosis inhibition was abolished by PD176252 (early and total AP: GRP+ PD 176252 vs. PD 176252 ; early AP: $9.59 \pm 0.43 \%$ vs. $9.62 \pm 0.41 \%$; total AP: $14.60 \pm 0.55 \%$ vs. $14.40 \pm 0.42 \%$; $\mathrm{P}>0.05$ ) and significantly compromised by U0126 (early and total AP: GRP+U0126 vs. U0126, early AP: $10.21 \pm 0.48 \%$ vs. $11.39 \pm 0.49 \%$, total AP: $15.40 \pm 0.53 \%$ vs. $16.56 \pm 0.58 \%$; $\mathrm{P}<0.05)$ (Fig. 7). Finally, GRP also markedly increased the percentages of cells in $\mathrm{S}$ phase and G2/M phase (GRP vs. $0.1 \%$ DMSO: S phase, $22.04 \pm 0.98 \%$ vs. $16.90 \pm 1.03 \%$; G2/M phase, $12.57 \pm 0.98 \%$ vs. $10.48 \pm 0.56 \%$; $\mathrm{P}<0.05)$ and reciprocally decreased the percentages of cells in G0/G1 phase (GRP vs. $0.1 \%$ DMSO: $65.38 \pm 2.13 \%$ vs. $76.12 \pm 2.12 \%$; $\mathrm{P}<0.05)$. The percentage of cells in $\mathrm{G} 0 / \mathrm{G} 1$ phase was significantly increased by PD176252 or U0126 $(\mathrm{P}<0.05)$. Concordantly, GRP-mediated stimulation of cell cycle progression was abolished by PD176252 (GRP+PD176252 vs. PD176252, G0/G1 phase, S phase, G2/M phase: $79.64 \pm 2.15 \%, 12.38 \pm 0.98 \%, 7.98 \pm 0.88 \%$ vs. $80.02 \pm 2.03 \%$, 
$12.42 \pm 0.56 \%, 7.56 \pm 0.89 \% ; \mathrm{P}>0.05)$ or significantly compromised by U0126 (GRP+U0126 vs. U0126, G0/G1 phase, S phase, G2/M phase: $84.09 \pm 2.05 \%, 9.21 \pm 1.02 \%, 7.22 \pm 0.96 \%$ vs. $86.21 \pm 2.18 \%, 7.51 \pm 0.69 \%, 6.28 \pm 0.98 \%$; P<0.05) (Fig. 8).

\section{Discussion}

GRP is an autocrine/paracrine growth factor for various human cancer cells (1-7). At present, several GRP analogues and GRPR antagonists are in preclinical trials for tumor imaging and anticancer therapy (8-14). Although it has been reported that GRP played a role in hepatocarcinogenesis in rat (19), no data are currently available confirming the contribution of GRP to human HCC. In the present study, we carried out pilot studies to examine the roles of GRP and GRPR in promoting the growth of HepG2 and MHCC97H human HCC cells and to delineate the possible mechanisms.

We first examined the expression of GRP and GRPR in normal hepatic cell line HL77-02 and human HCC cell lines HepG2 and MHCC97H. While both normal hepatic cell line and the two human HCC cell lines expressed detectable GRP and GRPR protein and mRNA, much higher levels of GRP and GRPR were detected in HepG2 and MHCC97H cells than in HL77-02 cells. These results indicated that GRP and GRPR were coexpressed in the two human HCC cell lines, consistent with previous observations with other tumor cells (3-7). Such a general coexpression pattern may suggest important biological effects, thus prompting us to further observe the influence of GRP on the growth of HepG2 cells with elevated GRP and GRPR expressions.

Our studies subsequently revealed enhanced stimulatory effects of GRP on the proliferation of HepG2 and MHCC97H cells compared with HL-7702 cells. Furthermore, the stimulatory role of GRP in the proliferation of HepG2 and $\mathrm{MHCC} 97 \mathrm{H}$ cells was dose- and time-dependent, with the maximum proliferation rate at the dose of $100 \mathrm{nM}$ and at the time-point of $48 \mathrm{~h}$. Moreover, we found that GRP transiently (within $8 \mathrm{~h}$ ) increased the levels of phosphorylated-ERK1/2 of HepG2 cells. The MAPK/ERK1/2 signaling pathway is a critically important signaling pathway promoting proliferation and migration of tumor cells, and activation of ERK1/2 in HepG2 cells might lead to the transcription of multiple genes required for growth and invasion $(21,22)$. Thus, our results are consistent with an important role of GRP in the growth of liver cancer cells as observed in other tumor cells (1-7,15-18).

In addition, we found that the GRP-induced activation of ERK1/2 was mainly mediated by GRPR overexpression in HepG2 cells; conversely, application of the GRPR antagonist PD176252 blocked the stimulatory effect of GRP on ERK1/2. Previously, GRP was found to interact with GRPR in tumor cells, consequently resulting in the activation of TACE. TACE belongs to the MMP (matrix metalloproteinase) super-family that function to modulate the growth and invasion of cancer cells through ECM (extracellular matrix) cleavage and activation of the MAPK/ERK1/2 signaling pathway by shedding ligands for EGFR $(17,18)$. However, we found that pre-incubation of HepG2 cells with the TACE inhibitor TAPI-1 or the EGFR tyrosine kinase inhibitor PD153035 did not diminish GRP-induced phosphorylation of ERK1/2. Thus, our data indicated that GRP activated ERK1/2 in
HepG2 cells through GRPR-dependent and TACE- and EGFR-independent mechanisms.

Finally, we demonstrated that GRP promoted the growth of HepG2 cells concurrently stimulating proliferation, inhibiting apoptosis and accelerating cell cycle progression. The stimulatory effect of GRP on tumor cell growth was mainly mediated by GRPR-dependent activation of ERK1/2. However, the MEK inhibitor U0126 did not completely abolish GRP-dependent enhancement of HepG2 cell growth, suggesting that ERK-independent mechanisms were also involved in mediating the effect of GRP on HepG2 cells.

In conclusion, GRP serves as a mitogen for HepG2 cells through interaction with GRPR, and subsequently activates the MAPK/ERK1/2 signaling pathway via EGFR-independent mechanisms.

\section{Acknowledgements}

We gratefully acknowledge Professor Chen Huang for valuable scientific discussions. We appreciate the assistance of Rongri Liang for cell culture and we thank Huanchen Sha for FCM analysis.

\section{References}

1. Patel O, Shulkes A and Baldwin GS: Gastrin-releasing peptide and cancer. Biochim Biophys Acta 1766: 23-41, 2006.

2. Qu X, Xiao D and Weber HC: Biologic relevance of mammalian bombesin-like peptides and their receptors in human malignancies. Curr Opin Endocrinology Diabetes 10: 60-71, 2003.

3. Hajri A, Balboni G, Koenig M, Garaud JC and Damge C: Gastrin-releasing peptide: in vivo and in vitro growth effects on an acinar pancreatic carcinoma. Cancer Res 52: 3726-3732, 1992.

4. Yano T, Pinski J, Groot K and Schally AV: Stimulation by bombesin and inhibition by bombesin/gastrin-releasing peptide antagonist RC-3095 of growth of human breast cancer cell lines. Cancer Res 52: 4545-4547, 1992.

5. Constantinides C, Lazaris AC, Haritopoulos KN, Pantazopoulos D, Chrisofos $\mathrm{M}$ and Giannopoulos A: Immunohistochemical detection of gastrin releasing peptide in patients with prostate cancer. World J Urol 21: 183-187, 2003.

6. Saurin JC, Rouault JP, Abello J, Berger F, Remy L and Chayvialle JA: High gastrin releasing peptide receptor mRNA level is related to tumour dedifferentiation and lymphatic vessel invasion in human colon cancer. Eur J Cancer 35: 125-132, 1999.

7. Fang MZ, Liu C, Song Y, et al: Overexpression of gastrinreleasing peptide in human esophageal squamous cell carcinomas. Carcinogenesis 25: 865-871, 2004.

8. Kelley MJ, Linnoila RI, Avis IL, et al: Antitumor activity of a monoclonal antibody directed against gastrin-releasing peptide in patients with small cell lung cancer. Chest 112: 256-261, 1997.

9. Cornelio DB, Roesler R and Schwartsmann G: Gastrin-releasing peptide receptor as a molecular target in experimental anticancer therapy. Ann Oncol 18: 1457-1466, 2007.

10. Zhou J, Chen J, Mokotoff M, Zhong R, Shultz LD and Ball ED: Bombesin/gastrin-releasing peptide receptor: a potential target for antibody-mediated therapy of small cell lung cancer. Clin Cancer Res 9: 4953-4960, 2003.

11. Qiao J, Kang J, Ishola TA, Rychahou PG, Evers BM and Chung DH: Gastrin-releasing peptide receptor silencing suppresses the tumorigenesis and metastatic potential of neuroblastoma. Proc Natl Acad Sci USA 105: 12891-12896, 2008.

12. Parry JJ, Andrews R and Rogers BE: MicroPET imaging of breast cancer using radiolabeled bombesin analogs targeting the gastrin-releasing peptide receptor. Breast Cancer Res Treat 101: 175-183, 2007.

13. Wiele CV, Phonteyne P, Pauwels P, et al: Gastrin-releasing peptide receptor imaging in human breast carcinoma versus immunohistochemistry. J Nucl Med 49: 260-264, 2008. 
14. SchweinsbergC, Maes V, Brans L, et al: Novel glycated [99mTc(CO)3]-labeled bombesin analogues for improved targeting of gastrin-releasing peptide receptor-positive tumors. Bioconjug Chem 19: 2432-2439, 2008.

15. Lui VW, Thomas SM, Zhang Q, et al: Mitogenic effects of gastrin-releasing peptide in head and neck squamous cancer cells are mediated by activation of the epidermal growth factor receptor. Oncogene 22: 6183-6193, 2003.

16. Chen PW and Kroog GS: Alterations in receptor expression or agonist concentration change the pathways gastrin-releasing peptide receptor uses to regulate extracellular signal-regulated kinase. Mol Pharmacol 166: 1625-1634, 2004.

17. Zhang Q, Thomas SM, Lui VW, et al: Phosphorylation of TNF- $\alpha$ converting enzyme by gastrin-releasing peptide induces amphiregulin release and EGF receptor activation. Proc Natl Acad Sci USA 103: 6901-6906, 2006.

18. Thomas SM, Grandis JR, Wentzel AL, Gooding WE, Lui VW and Siegfried JM: Gastrin-releasing peptide receptor mediates activation of the epidermal growth factor receptor in lung cancer cells. Neoplasia 7: 426-431, 2005.
19. Seglen PO, Skomedal H, Saeter G, Schwarze PE and Nesland JM: Neuroendocrine dysdifferentiation and bombesin production in carcinogen-induced hepatocellular rat tumours. Carcinogenesis 10: 21-25, 1989 .

20. Breuhahn K, Longerich T and Schirmacher P: Dysregulation of growth factor signaling in human hepatocellular carcinoma. Oncogene 25: 3787-3800, 2006.

21. Tsuboi Y, Ichida T, Sugitani S, et al: Overexpression of extracellular signal-regulated protein kinase and its correlation with proliferation in human hepatocellular carcinoma. Liver Int 24: 432-436, 2004.

22. Schmitz KJ, Wohlschlaeger J, Lang $\mathrm{H}$, et al: Activation of the ERK and AKT signalling pathway predicts poor prognosis in hepatocellular carcinoma and ERK activation in cancer tissue is associated with hepatitis C virus infection. J Hepatol 48: 83-90, 2008. 